Article

\title{
Some Identities of Fully Degenerate Bernoulli Polynomials Associated with Degenerate Bernstein Polynomials
}

\author{
Jeong Gon Lee ${ }^{1}$, Wonjoo Kim ${ }^{2, *}$ and Lee-Chae Jang ${ }^{3}$ \\ 1 Division of Applied Mathematics, Nanoscale Science and Technology Institute, Wonkwang University, \\ Iksan 54538, Korea; jukolee@wku.ac.kr \\ 2 Department of Applied Mathematics, Kyunghee University, Yongin 17104, Korea \\ 3 Graduate School of Education, Konkuk University, Seoul 05029, Korea; lcjang@konkuk.ac.kr \\ * Correspondence: wjookim@khu.ac.kr
}

Received: 26 April 2019; Accepted: 20 May 2019; Published: 24 May 2019

check for

Abstract: In this paper, we investigate some properties and identities for fully degenerate Bernoulli polynomials in connection with degenerate Bernstein polynomials by means of bosonic $p$-adic integrals on $\mathbb{Z}_{p}$ and generating functions. Furthermore, we study two variable degenerate Bernstein polynomials and the degenerate Bernstein operators.

Keywords: degenerate Bernoulli polynomials; degenerate Bernstein operators

\section{Introduction}

Let $p$ be a fixed prime number. Throughout this paper, $\mathbb{Z}, \mathbb{Z}_{p}, \mathbb{Q}_{p}$ and $\mathbb{C}_{p}$, will denote the ring of rational integers, the ring of $p$-adic integers, the field of $p$-adic rational numbers and the completion of algebraic closure of $\mathbb{Q}_{p}$, respectively. The $p$-adic norm $|q|_{p}$ is normalized as $|p|_{p}=\frac{1}{p}$.

For $\lambda, t \in \mathbb{C}_{p}$ with $|\lambda t|_{p}<p^{-\frac{1}{p-1}}$ and $|t|_{p}<1$, the degenerate Bernoulli polynomials are defined by the generating function to be

$$
\frac{t}{(1+\lambda t)^{\frac{1}{\lambda}}-1}(1+\lambda t)^{\frac{x}{\lambda}}=\sum_{n=0}^{\infty} \beta_{n}(x \mid \lambda) \frac{t^{n}}{n !},
$$

(See [1-3]). When $x=0, \beta_{n}(\lambda)=\beta_{n}(0 \mid \lambda)$ are called the degenerate Bernoulli numbers. Note that $\lim _{\lambda \rightarrow 0} \beta_{n}(x \mid \lambda)=B_{n}(x)$, where $B_{n}(x)$ are the ordinary Bernoulli polynomials defined by

$$
\frac{t}{e^{t}-1} e^{x t}=\sum_{n=0}^{\infty} B_{n}(x) \frac{t^{n}}{n !}
$$

and $B_{n}=B_{n}(0)$ are called the Bernoulli numbers. The degenerate exponential function is defined by

$$
e_{\lambda}^{x}(t)=(1+\lambda t)^{\frac{x}{\lambda}}=\sum_{n=0}^{\infty}(x)_{n, \lambda} \frac{t^{n}}{n !},
$$

where $(x)_{0, \lambda}=1,(x)_{n, \lambda}=x(x-\lambda)(x-2 \lambda) \cdots(x-(n-1) \lambda)$, for $n \geq 1$. From (1), we get

$$
\beta_{n}(x \mid \lambda)=\sum_{l=0}^{n}\left(\begin{array}{l}
n \\
l
\end{array}\right) \beta_{l}(\lambda)(x)_{n-l, \lambda} .
$$


Recentely, Kim-Kim introduced the degenerate Bernstein polynomials given by

$$
\frac{(x)_{k, \lambda}}{k !} t^{k}(1+\lambda t)^{\frac{1-x}{\lambda}}=\sum_{n=k}^{\infty} B_{k, n}(x \mid \lambda) \frac{t^{n}}{n !},
$$

(See [4-6]). Thus, by (5), we note that

$$
B_{k, n}(x \mid \lambda)= \begin{cases}\left(\begin{array}{c}
n \\
k
\end{array}\right)(x)_{k, \lambda}(1-x)_{n-k, \lambda}, & \text { if } n \geq k \\
0, & \text { if } n<k\end{cases}
$$

where $n, k$ are non-negative integers. Let $U D\left(\mathbb{Z}_{p}\right)$ be the space of uniformly differentiable functions on $\mathbb{Z}_{p}$. For $f \in U D\left(\mathbb{Z}_{p}\right)$, the degenerate Bernstein operator of order $n$ is given by

$$
\begin{aligned}
\mathbb{B}_{n, \lambda}(f \mid \lambda)= & \sum_{k=0}^{\infty} f\left(\frac{k}{n}\right)\left(\begin{array}{l}
n \\
k
\end{array}\right)(x)_{k, \lambda}(1-x)_{n-k, \lambda} \\
& =\sum_{k=0}^{\infty} f\left(\frac{k}{n}\right) B_{k, n}(x \mid \lambda)
\end{aligned}
$$

(See [4-6]). The bosonic $p$-adic integral on $\mathbb{Z}_{p}$ is defined by Volkenborn as

$$
\int_{\mathbb{Z}_{p}} f(x) d \mu_{0}(x)=\lim _{N \rightarrow \infty} \frac{1}{p^{N}} \sum_{x=0}^{p^{N}-1} f(x),
$$

(see [7]). By (8), we get

$$
\int_{\mathbb{Z}_{p}} f(x+1) d \mu_{0}(x)-\int_{\mathbb{Z}_{p}} f(x) d \mu_{0}(x)=f^{\prime}(0),
$$

where $\left.\frac{d}{d x} f(x)\right|_{x=0}=f^{\prime}(0)$.

From (8), Kim-Seo [8] proposed fully degenerate Bernoulli polynomials which are reformulated in terms of bosonic $p$-adic integral on $\mathbb{Z}_{p}$ as

$$
\int_{\mathbb{Z}_{p}}(1+\lambda t)^{\frac{x+y}{\lambda}} d \mu_{0}(y)=\frac{\frac{1}{\lambda} \log (1+\lambda t)}{(1+\lambda t)^{\frac{1}{\lambda}}-1}(1+\lambda t)^{\frac{x}{\lambda}}=\sum_{n=0}^{\infty} B_{n}(x \mid \lambda) \frac{t^{n}}{n !},
$$

and for $x=0, B_{n}(\lambda)=B_{n}(0 \mid \lambda)$ are called fully degenerate Bernoulli numbers.

Note that the fully degenerate Bernoulli polynomial was named Daehee polynomials with $\alpha$-parameter in [9]. On the other hand,

$$
\int_{\mathbb{Z}_{p}}(1+\lambda t)^{\frac{x+y}{\lambda}} d \mu_{0}(y)=\sum_{n=0}^{\infty} \int_{\mathbb{Z}_{p}}(x+y)_{n, \lambda} d \mu_{0}(y) \frac{t^{n}}{n !} .
$$

By (10) and (11), we get

$$
\int_{\mathbb{Z}_{p}}(x+y)_{n, \lambda} d \mu_{0}(y)=B_{n}(x \mid \lambda), \quad(n \geq 0) .
$$

Recall that the Daehee polynomials are defined by the generating function to be

$$
\frac{\log (1+t)}{t}(1+t)^{x}=\sum_{n=0}^{\infty} D_{n}(x) \frac{t^{n}}{n !},
$$

and for $x=0, D_{n}=D_{n}(0)$ are called the Daehee numbers (see $[10,11]$ ). 
Also, the higher order Daehee polynomials are defined by the generating function to be

$$
\left(\frac{\log (1+t)}{t}\right)^{k}(1+t)^{x}=\sum_{n=0}^{\infty} D_{n}^{(k)}(x) \frac{t^{n}}{n !}
$$

and for $x=0, D_{n}^{(k)}=D_{n}^{(k)}(0)$ are called the higher order Daehee numbers. From (10), we observe

$$
\begin{aligned}
\frac{\frac{1}{\lambda} \log (1+\lambda t)}{(1+\lambda t)^{\frac{1}{\lambda}}-1}(1+\lambda t)^{\frac{x}{\lambda}} & =\frac{t}{(1+\lambda t)^{\frac{1}{\lambda}}-1}(1+\lambda t)^{\frac{x}{\lambda}} \frac{\log (1+\lambda t)}{\lambda t} \\
& =\left(\sum_{m=0}^{\infty} \beta_{m}(x \mid \lambda) \frac{t^{m}}{m !}\right)\left(\sum_{l=0}^{\infty} D_{l} \frac{(\lambda t)^{l}}{l !}\right) \\
& =\sum_{n=0}^{\infty}\left(\sum_{m=0}^{n}\left(\begin{array}{c}
n \\
m
\end{array}\right) \beta_{m}(x \mid \lambda) D_{n-m} \lambda^{n-m}\right) \frac{t^{n}}{n !} .
\end{aligned}
$$

By (10) and (14), we get

$$
B_{n}(x \mid \lambda)=\sum_{m=0}^{n}\left(\begin{array}{c}
n \\
m
\end{array}\right) \beta_{m}(x \mid \lambda) D_{n-m} \lambda^{n-m}, \quad(n \geq 0) .
$$

From (3) and (10), we observe that

$$
\begin{aligned}
\sum_{n=0}^{\infty} B_{n}(x \mid \lambda) \frac{t^{n}}{n !} & =\frac{\frac{1}{\lambda} \log (1+\lambda t)}{(1+\lambda t)^{\frac{1}{\lambda}}-1}(1+\lambda t)^{\frac{x}{\lambda}} \\
& =\left(\sum_{m=0}^{\infty} B_{m}(\lambda) \frac{t^{m}}{m !}\right)\left(\sum_{l=0}^{\infty}(x)_{l, \lambda} \frac{t^{l}}{l !}\right) \\
& =\sum_{n=0}^{\infty}\left(\sum_{m=0}^{n}\left(\begin{array}{c}
n \\
m
\end{array}\right) B_{m}(\lambda)(x)_{n-m, \lambda}\right) \frac{t^{n}}{n !} .
\end{aligned}
$$

By (17), we get

$$
B_{n}(x \mid \lambda)=\sum_{m=0}^{n}\left(\begin{array}{c}
n \\
m
\end{array}\right) B_{m}(\lambda)(x)_{n-m, \lambda}, \quad(n \geq 0) .
$$

From (1) and (3), we note that

$$
\begin{aligned}
t & =\left((1+\lambda t)^{\frac{1}{\lambda}}-1\right) \sum_{m=0}^{\infty} \beta_{m}(\lambda) \frac{t^{m}}{m !} \\
& =\left(\sum_{l=0}^{\infty}(1)_{l, \lambda} \frac{t^{l}}{l !}\right)\left(\sum_{m=0}^{\infty} \beta_{m}(\lambda) \frac{t^{m}}{m !}\right)-\sum_{m=0}^{\infty} \beta_{m}(\lambda) \frac{t^{m}}{m !} \\
& =\sum_{n=0}^{\infty}\left(\sum_{m=0}^{n}\left(\begin{array}{c}
n \\
m
\end{array}\right)(1)_{n-m, \lambda} \beta_{m}(\lambda)\right) \frac{t^{n}}{n !}-\sum_{n=0}^{\infty} \beta_{n}(\lambda) \frac{t^{n}}{n !} \\
& =\sum_{n=0}^{\infty}\left(\sum_{m=0}^{n}\left(\begin{array}{c}
n \\
m
\end{array}\right)(1)_{n-m, \lambda} \beta_{m}(\lambda)-\beta_{n}(\lambda)\right) \frac{t^{n}}{n !} .
\end{aligned}
$$

Comparing the cofficients on both sides of (19), we get

$$
\sum_{m=0}^{n}\left(\begin{array}{c}
n \\
m
\end{array}\right)(1)_{n-m, \lambda} \beta_{m}(\lambda)-\beta_{n}(\lambda)=\delta_{1, n}, \quad(n \geq 0),
$$

where $\delta_{k, n}$ is the Kronecker's symbol. 
By (4) and (20), we have

$$
\beta_{n}(1 \mid \lambda)-\beta_{n}(\lambda)=\delta_{1, n} .
$$

The generating function of fully degenerate Bernoulli polynomials introduced in (5) can be expressed as bosonic $p$-adic integral but the generating function of degenerate Bernoulli polynomials introduced in (1) is not expressed as a bosonic $p$-adic integral. This is why we considered the fully degenerate Bernoulli polynomials, and the motivation of this paper is to investigate some identities of them associated with degenerate Bernstein polynomials.

In this paper, we consider the fully degenerate Bernoulli polynomials and investigate some properties and identities for these polynomials in connection with degenerate Bernstein polynomials by means of bosonic $p$-adic integrals on $\mathbb{Z}_{p}$ and generating functions. Furthermore, we study two variable degenerate Bernstein polynomials and the degenerate Bernstein operators.

\section{Fully Degenerate Bernoulli and Bernstein Polynomials}

From (10), we observe that

$$
\begin{aligned}
\sum_{n=0}^{\infty} B_{n}(1-x \mid \lambda) \frac{t^{n}}{n !} & =\frac{\frac{1}{\lambda} \log (1+\lambda t)}{(1+\lambda t)^{\frac{1}{\lambda}}-1}(1+\lambda t)^{\frac{1-x}{\lambda}} \\
& =\frac{\left(-\frac{1}{\lambda}\right)(1+(-\lambda)(-t))}{(1+(-\lambda)(-t))^{-\frac{1}{\lambda}}-1}(1+(-\lambda)(-t))^{-\frac{x}{\lambda}} \\
& =\sum_{n=0}^{\infty} B_{n}(x \mid-\lambda)(-1)^{n} \frac{t^{n}}{n !}
\end{aligned}
$$

From (22), we obtain the following Lemma.

Lemma 1. For $n \in \mathbb{N} \cup\{0\}$, we have

$$
B_{n}(1-x \mid \lambda)=(-1)^{n} B_{n}(x \mid-\lambda) .
$$

From (16) and (21), we get

$$
\begin{aligned}
B_{n}(1 \mid \lambda)-B_{n}(\lambda)= & \sum_{m=0}^{n}\left(\begin{array}{c}
n \\
m
\end{array}\right)\left(\beta_{m}(1 \mid \lambda)-\beta_{m}(\lambda)\right) D_{n-m} \lambda^{n-m} \\
& =\sum_{m=0}^{n}\left(\begin{array}{c}
n \\
m
\end{array}\right) \delta_{1, m} D_{n-m} \lambda^{n-m}, \quad(n \geq 0) .
\end{aligned}
$$

From (1), we observe that

$$
\begin{aligned}
\sum_{n=0}^{\infty} \beta_{n}(x+1 \mid \lambda) \frac{t^{n}}{n !} & =(1+\lambda t)^{\frac{1}{\lambda}}\left(\sum_{m=0}^{\infty} \beta_{m}(x \mid \lambda) \frac{t^{m}}{m !}\right) \\
& =\left(\sum_{l=0}^{\infty}(1)_{l, \lambda} \frac{t^{l}}{l !}\right)\left(\sum_{m=0}^{\infty} \beta_{m}(x \mid \lambda) \frac{t^{m}}{m !}\right) \\
& =\sum_{n=0}^{\infty}\left(\sum_{m=0}^{n}\left(\begin{array}{c}
n \\
m
\end{array}\right)(1)_{n-m, \lambda} \beta_{m}(x \mid \lambda)\right) \frac{t^{n}}{n !} .
\end{aligned}
$$

By (25), we get

$$
\beta_{n}(x+1 \mid \lambda)=\sum_{m=0}^{n}\left(\begin{array}{c}
n \\
m
\end{array}\right)(1)_{n-m, \lambda} \beta_{m}(x \mid \lambda) .
$$


By (26), with $x=1$, we have

$$
\begin{aligned}
\beta_{n}(2 \mid \lambda) & =\sum_{m=0}^{n}\left(\begin{array}{c}
n \\
m
\end{array}\right) \beta_{m}(1 \mid \lambda)(1)_{n-m, \lambda} \\
& =(1)_{n, \lambda} \beta_{0}(1 \mid \lambda)+n(1)_{n-1, \lambda} \beta_{1}(1 \mid \lambda)+\sum_{m=2}^{n}\left(\begin{array}{c}
n \\
m
\end{array}\right) \beta_{m}(1 \mid \lambda)(1)_{n-m, \lambda} \\
& =(1)_{n, \lambda}+n(1)_{n-1, \lambda}\left(\beta_{1}(\lambda)-1\right)+\sum_{m=2}^{n}\left(\begin{array}{c}
n \\
m
\end{array}\right) \beta_{m}(\lambda)(1)_{n-m, \lambda} \\
& =(1)_{n, \lambda}+n(1)_{n-1, \lambda} \beta_{1}(\lambda)-n(1)_{n-1, \lambda}+\sum_{m=2}^{n}\left(\begin{array}{c}
n \\
m
\end{array}\right) \beta_{m}(\lambda)(1)_{n-m, \lambda} \\
& =-n(1)_{n-1, \lambda}+\sum_{m=0}^{n}\left(\begin{array}{c}
n \\
m
\end{array}\right) \beta_{m}(\lambda)(1)_{n-m, \lambda} \\
& =-n(1)_{n-1, \lambda}+\beta_{n}(1 \mid \lambda) .
\end{aligned}
$$

Therefore, by (27), we obtain the following theorem.

Theorem 1. For $n \in \mathbb{N}$, we have

$$
\beta_{n}(2 \mid \lambda)=-n(1)_{n-1, \lambda}+\beta_{n}(1 \mid \lambda)
$$

Note that

$$
(1-x)_{n, \lambda}=(-1)^{n}(x-1)_{n,-\lambda,} \quad(n \geq 0) .
$$

Therefore by (12), (23), and (29), we get

$$
\int_{\mathbb{Z}_{p}}(1-x)_{n, \lambda} d \mu_{0}(x)=(-1)^{n} \int_{\mathbb{Z}_{p}}(x-1)_{n,-\lambda} d \mu_{0}(x)=\int_{\mathbb{Z}_{p}}(x+2)_{n, \lambda} d \mu_{0}(x) .
$$

Therefore, by (30) and Theorem 1, we obtain the following theorem.

Theorem 2. For $n \in \mathbb{N}$, we have

$$
\int_{\mathbb{Z}_{p}}(1-x)_{n, \lambda} d \mu_{0}(x)=\int_{\mathbb{Z}_{p}}(x+2)_{n, \lambda} d \mu_{0}(x)=n(1)_{n-1, \lambda}(\lambda-1) B_{1}(\lambda)+\int_{\mathbb{Z}_{p}}(x)_{n, \lambda} d \mu_{0}(x) .
$$

Corollary 1. For $n \in \mathbb{N}$, we have

$$
(-1)^{n} B_{n}(-1 \mid-\lambda)=(1)_{n-1, \lambda}\left(1-n B_{1}(\lambda)\right)+B_{n}(\lambda)=B_{n}(2 \mid \lambda) .
$$

By (17), we get

$$
\begin{aligned}
B_{n}(1-x \mid \lambda) & =\sum_{m=0}^{n}\left(\begin{array}{c}
n \\
m
\end{array}\right) B_{m}(\lambda)(1-x)_{n-m, \lambda} \\
& =\sum_{m=0}^{n}\left(\begin{array}{c}
n \\
m
\end{array}\right)(x)_{m, \lambda}(1-x)_{n-m, \lambda} \frac{B_{m}(\lambda)}{(x)_{m, \lambda}} \\
& =\sum_{m=0}^{n} B_{m, n}(x \mid \lambda) B_{m}(\lambda) \frac{1}{(x)_{m, \lambda}}
\end{aligned}
$$


In [8], we note that

$$
\begin{aligned}
\frac{1}{(x)_{m, \lambda}} & =\frac{1}{x(x-\lambda)(x-2 \lambda) \cdots(x-(m-1) \lambda)} \\
& =\sum_{k=0}^{m-1} \frac{(-1)^{k}}{(m-1) !}\left(\begin{array}{c}
m-1 \\
k
\end{array}\right) \frac{(-\lambda)^{1-m}}{x-k \lambda}, \quad(m \in \mathbb{N}) .
\end{aligned}
$$

By (33) and (34) we get

$$
\begin{aligned}
B_{n}(1-x \mid \lambda) & =\sum_{m=0}^{n} B_{m, n}(x \mid \lambda) B_{m}(\lambda) \frac{1}{(x)_{m, \lambda}} \\
& =(1-x)_{n, \lambda}+\sum_{m=1}^{n} B_{m, n}(x \mid \lambda) B_{m}(\lambda) \frac{1}{(x)_{m, \lambda}} \\
& =(1-x)_{n, \lambda}+\sum_{m=1}^{n} B_{m, n}(x \mid \lambda) B_{m}(\lambda) \frac{(-\lambda)^{1-m}}{(m-1) !} \sum_{k=0}^{m-1}(-1)^{k}\left(\begin{array}{c}
m-1 \\
k
\end{array}\right) \frac{1}{x-k \lambda} .
\end{aligned}
$$

Therefore, by (35), we obtain the following theorem.

Theorem 3. For $n \in \mathbb{N} \cup\{0\}$, we have

$$
B_{n}(1-x \mid \lambda)=(1-x)_{n, \lambda}+\sum_{m=1}^{n} B_{m, n}(x \mid \lambda) B_{m}(\lambda) \frac{(-\lambda)^{1-m}}{(m-1) !} \sum_{k=0}^{m-1}(-1)^{k}\left(\begin{array}{c}
m-1 \\
k
\end{array}\right) \frac{1}{x-k \lambda} .
$$

Corollary 2. For $n \in \mathbb{N} \cup\{0\}$, we have

$$
B_{n}(2 \mid \lambda)=(2)_{n, \lambda}+\sum_{m=1}^{n} B_{m, n}(-1 \mid \lambda) B_{m}(\lambda) \frac{(-\lambda)^{1-m}}{(m-1) !} \sum_{k=0}^{m-1}(-1)^{k+1}\left(\begin{array}{c}
m-1 \\
k
\end{array}\right) \frac{1}{1+k \lambda} .
$$

For $k \in \mathbb{N}$, the higher-order fully degenerate Bernoulli polynomials are given by the generating function

$$
\left(\frac{\frac{1}{\lambda} \log (1+\lambda t)}{(1+\lambda t)^{\frac{1}{\lambda}}-1}\right)^{k}(1+\lambda t)^{\frac{x}{\lambda}}=\sum_{n=0}^{\infty} B_{n}^{(k)}(x \mid \lambda) \frac{t^{n}}{n !},
$$

(See $[8,12,13])$. When $x=0, B_{n}^{(k)}(\lambda)=B_{n}^{(k)}(x \mid 0)$ are called the higher-order fully degenerate Bernoulli numbers. From (5) and (38), we note that

$$
\begin{aligned}
\left(\frac{\log (1+\lambda t)}{\lambda t}\right)^{k} \sum_{n=k}^{\infty} B_{k, n}(x \mid \lambda) \frac{t^{n}}{n !} & =(x)_{k, \lambda} t^{k}(1+\lambda t)^{\frac{1-x}{\lambda}}\left(\frac{\log (1+\lambda t)}{\lambda t}\right)^{k} \frac{1}{k !} \\
& =\frac{\left((1+\lambda t)^{\frac{1}{\lambda}}-1\right)^{k}}{\left((1+\lambda t)^{\frac{1}{\lambda}}-1\right)^{k}}(x)_{k, \lambda}\left(\frac{1}{\lambda} \log (1+\lambda t)\right)^{k}(1+\lambda t)^{\frac{1-x}{\lambda}} \frac{1}{k !} \\
& =(x)_{k, \lambda} \sum_{m=0}^{k}\left(\begin{array}{c}
k \\
m
\end{array}\right)(-1)^{m-k}(1+\lambda t)^{\frac{m}{\lambda}}\left(\frac{\frac{1}{\lambda} \log (1+\lambda t)}{(1+\lambda t)^{\frac{1}{\lambda}}-1}\right)^{k}(1+\lambda t)^{\frac{1-x}{\lambda}} \frac{1}{k !} \\
& =(x)_{k, \lambda} \sum_{m=0}^{k}\left(\begin{array}{c}
k \\
m
\end{array}\right)(-1)^{m-k}\left(\frac{\frac{1}{\lambda} \log (1+\lambda t)}{(1+\lambda t)^{\frac{1}{\lambda}}-1}\right)^{k}(1+\lambda t)^{\frac{1-x+m}{\lambda}} \frac{1}{k !} \\
& =(x)_{k, \lambda} \sum_{m=0}^{k}\left(\begin{array}{c}
k \\
m
\end{array}\right)(-1)^{m-k} \sum_{n=0}^{\infty} B_{n}(1-x+m \mid \lambda) \frac{t^{n}}{n !} \frac{1}{k !} \\
& =\sum_{n=0}^{\infty}\left((x)_{k, \lambda} \sum_{m=0}^{k}\left(\begin{array}{c}
k \\
m
\end{array}\right)(-1)^{m-k} B_{n}(1-x+m \mid \lambda) \frac{1}{k !}\right) \frac{t^{n}}{n !}
\end{aligned}
$$


and hence, we get

$$
\begin{aligned}
\left(\frac{\log (1+\lambda t)}{\lambda t}\right)^{k} \sum_{m=k}^{\infty} B_{k, m}(x \mid \lambda) \frac{t^{m}}{m !} & =\left(\sum_{l=0}^{\infty} D_{l}^{(k)} \lambda^{l} \frac{t^{l}}{l !}\right)\left(\sum_{m=k}^{\infty} B_{k, m}(x \mid \lambda) \frac{t^{m}}{m !}\right) \\
& =\sum_{n=k}^{\infty}\left(\sum_{l=0}^{n} D_{l}^{(k)} \lambda^{l} B_{k, n-l}(x \mid \lambda)\right) \frac{t^{n}}{n !} .
\end{aligned}
$$

Therefore, by (39) and (40), we obtain the following theorem.

Theorem 4. For $k, n \in \mathbb{N}$, we have

$$
\frac{1}{k !}(x)_{n, \lambda} \sum_{m=0}^{k}\left(\begin{array}{c}
k \\
m
\end{array}\right)(-1)^{m-k} B_{n}(1-x+m \mid \lambda)= \begin{cases}\sum_{l=0}^{n} D_{l}^{(k)} \lambda^{l} B_{k, n-l}(x \mid \lambda), & \text { if } n \geq k \\
0, & \text { if } n<k\end{cases}
$$

Let $f \in U D\left(\mathbb{Z}_{p}\right)$. For $x_{1}, x_{2} \in \mathbb{Z}_{p}$, we consider the degenerate Bernstein operator of order $n$ given by

$$
\mathbb{B}_{n, \lambda}\left(f \mid x_{1}, x_{2}\right)=\sum_{k=0}^{n} f\left(\frac{k}{n}\right)\left(\begin{array}{l}
n \\
k
\end{array}\right)\left(x_{1}\right)_{k, \lambda}\left(1-x_{2}\right)_{n-k, \lambda}=\sum_{k=0}^{n} f\left(\frac{k}{n}\right) B_{k, n}\left(x_{1}, x_{2} \mid \lambda\right),
$$

where $B_{n, k}\left(x_{1}, x_{2} \mid \lambda\right)$ are called two variable degenerate Bernstein polynomials of degree $n$ as followings (see, $[2-6,9,14-27])$ :

$$
B_{k, n}\left(x_{1}, x_{2} \mid \lambda\right)=\left(\begin{array}{l}
n \\
k
\end{array}\right)\left(x_{1}\right)_{k, \lambda}\left(1-x_{2}\right)_{n-k, \lambda,}(n \geq 0) .
$$

The authors [3] obtained the following:

$$
\sum_{k=0}^{\infty} B_{k, n}\left(x_{1}, x_{2} \mid \lambda\right) \frac{t^{n}}{n !}=\frac{\left(x_{1}\right)_{k, \lambda}}{k !} t^{k} e_{\lambda}^{1-x_{2}}(t)
$$

The authors [8] obtained the following:

$$
\begin{aligned}
B_{k, n}\left(x_{1}, x_{2} \mid \lambda\right) & =\left(\begin{array}{l}
n \\
k
\end{array}\right)\left(1-\left(1-x_{1}\right)\right)_{n-(n-k), \lambda}\left(1-x_{2}\right)_{n-k, \lambda} \\
& =B_{n-k, n}\left(1-x_{2}, 1-x_{1} \mid \lambda\right),
\end{aligned}
$$

and

$$
\begin{aligned}
B_{k, n}\left(x_{1}, x_{2} \mid \lambda\right)= & \left(1-x_{2}-(n-k-1) \lambda\right) B_{k, n-1}\left(x_{1}, x_{2} \mid \lambda\right) \\
& +\left(x_{1}-(k-1) \lambda\right) B_{k-1, n-1}\left(x_{1}, x_{2} \mid \lambda\right) .
\end{aligned}
$$

From (42), we note that $x_{1}, x_{2} \in \mathbb{Z}_{p}$, if $f(x)=1$, then we have

$$
\begin{aligned}
\mathbb{B}_{n, \lambda}\left(1 \mid x_{1}, x_{2}\right) & =\sum_{k=0}^{n} B_{k, n}\left(x_{1}, x_{2} \mid \lambda\right) \\
& =\sum_{k=0}^{n}\left(\begin{array}{l}
n \\
k
\end{array}\right)\left(x_{1}\right)_{k, \lambda}\left(1-x_{2}\right)_{n-k, \lambda} \\
& =\left(1+x_{1}-x_{2}\right)_{n, \lambda},
\end{aligned}
$$

and if $f(t)=t$, then we have

$$
\mathbb{B}_{n, \lambda}\left(t \mid x_{1}, x_{2}\right)=\left(x_{1}\right)_{1, \lambda}\left(x_{1}+1-\lambda-x_{2}\right)_{n-1, \lambda},
$$

and if $f(t)=t^{2}$, then we have 
$\mathbb{B}_{n, \lambda}\left(t^{2} \mid x_{1}, x_{2}\right)=\frac{1}{n}\left(x_{1}\right)_{1, \lambda}\left(x_{1}+1-\lambda-x_{2}\right)_{n-1, \lambda}+\frac{n-1}{n}\left(x_{1}\right)_{2, \lambda}\left(1+x_{2}-2 \lambda-x_{2}\right)_{n-2, \lambda}$.

The authors [3] obtained the following:

$$
(x)_{1, \lambda}=\frac{1}{\left(x_{1}+1-\lambda-x_{2}\right)_{n-1, \lambda}} \mathbb{B}_{n}\left(t \mid x_{1}, x_{2}\right),
$$

and

$$
(x)_{2, \lambda}=\frac{1}{\left(x_{1}+1-2 \lambda-x_{2}\right)_{n-2, \lambda}} \sum_{k=2}^{n} \frac{\left(\begin{array}{c}
k \\
2
\end{array}\right)}{\left(\begin{array}{c}
n \\
2
\end{array}\right)} B_{k, n}\left(x_{1}, x_{2} \mid \lambda\right),
$$

and

$$
(x)_{i, \lambda}=\frac{1}{\left(1+x_{1}-x_{2}-i \lambda\right)_{n-i, \lambda}} \sum_{k=i}^{n} \frac{\left(\begin{array}{c}
k \\
i
\end{array}\right)}{\left(\begin{array}{c}
n \\
i
\end{array}\right)} B_{k, n}\left(x_{1}, x_{2} \mid \lambda\right) .
$$

Taking double bosonic $p$-adic integral on $\mathbb{Z}_{p}$, we get the following equation:

$$
\int_{\mathbb{Z}_{p}} \int_{\mathbb{Z}_{p}} B_{k, n}\left(x_{1}, x_{2} \mid \lambda\right) d \mu_{0}\left(x_{1}\right) d \mu_{0}\left(x_{2}\right)=\left(\begin{array}{l}
n \\
k
\end{array}\right) \int_{\mathbb{Z}_{p}}\left(x_{1}\right)_{k, \lambda} d \mu_{0}\left(x_{1}\right) \int_{\mathbb{Z}_{p}}\left(1-x_{2}\right)_{n-k, \lambda} d \mu_{0}\left(x_{2}\right) .
$$

Therefore, by (53) and Theorem 2, we obtain the following theorem.

Theorem 5. For $n, k \in \mathbb{N} \cup\{0\}$, we have

$$
\begin{aligned}
& \int_{\mathbb{Z}_{p}} \int_{\mathbb{Z}_{p}} B_{k, n}\left(x_{1}, x_{2} \mid \lambda\right) d \mu_{0}\left(x_{1}\right) d \mu_{0}\left(x_{2}\right) \\
& = \begin{cases}\left(\begin{array}{l}
n \\
k
\end{array}\right) B_{n}(\lambda)\left((1)_{n-1, \lambda} n(\lambda-1) B_{n}(\lambda)+B_{n-k}(\lambda)\right), & \text { if } n>k, \\
B_{n}(\lambda), & \text { if } n=k .\end{cases}
\end{aligned}
$$

We get from the symmetric properties of two variable degenerate Bernstein polynomials that for $n, k \in \mathbb{N}$ with $n>k$,

$$
\begin{aligned}
& \int_{\mathbb{Z}_{p}} \int_{\mathbb{Z}_{p}} B_{k, n}\left(x_{1}, x_{2} \mid \lambda\right) d \mu_{0}\left(x_{1}\right) d \mu_{0}\left(x_{2}\right) \\
& =\sum_{m=0}^{k}\left(\begin{array}{l}
n \\
k
\end{array}\right)\left(\begin{array}{l}
k \\
m
\end{array}\right)(-1)^{k+m}(1)_{m, \lambda} \\
& \times \int_{\mathbb{Z}_{p}} \int_{\mathbb{Z}_{p}}\left(1-x_{1}\right)_{k-m,-\lambda}\left(1-x_{2}\right)_{n-k, \lambda} d \mu_{0}\left(x_{1}\right) d \mu_{0}\left(x_{2}\right) \\
& =\left(\begin{array}{l}
n \\
k
\end{array}\right) \int_{\mathbb{Z}_{p}}\left(1-x_{2}\right)_{n-k} d \mu_{0}\left(x_{2}\right) \sum_{m=0}^{k}\left(\begin{array}{l}
n \\
k
\end{array}\right)\left(\begin{array}{l}
k \\
m
\end{array}\right)(-1)^{k+m}(1)_{m, \lambda} d \mu_{0}\left(x_{2}\right) \\
& \times\left\{(1)_{k-m,-\lambda}(k-m)(-\lambda-1) B_{1}(-\lambda)+\int_{\mathbb{Z}_{p}}\left(x_{1}\right)_{k-m,-\lambda} d \mu_{0}\left(x_{1}\right)\right\} \\
& =\left(\begin{array}{l}
n \\
k
\end{array}\right) B_{n-k, \lambda}(2) \sum_{m=0}^{k}\left(\begin{array}{l}
n \\
k
\end{array}\right)\left(\begin{array}{c}
k \\
m
\end{array}\right)(-1)^{k+m}(1)_{m, \lambda} \\
& \times\left\{(1)_{k-m,-\lambda}(k-m)(-\lambda-1) B_{1}(-\lambda)+B_{k-m,-\lambda}(2)\right\}
\end{aligned}
$$

Therefore, by Theorem 5 , we obtain the following theorem. 
Theorem 6. For $n, k \in \mathbb{N} \cup\{0\}$, we have the following identities:

1. If $n>k$, then we have

$$
\begin{aligned}
B_{n}\left((1)_{n-1, \lambda} n(\lambda-1) B_{1}(\lambda)+B_{n-k}(\lambda)\right) \\
=B_{n-k, \lambda}(2) \sum_{m=0}^{k}\left(\begin{array}{l}
n \\
k
\end{array}\right)\left(\begin{array}{c}
k \\
m
\end{array}\right)(-1)^{k+m}(1)_{m, \lambda} \\
\quad \times\left((1)_{k-m,-\lambda}(k-m)(-\lambda-1) B_{1}(-\lambda)+B_{k-m,-\lambda}(2)\right) .
\end{aligned}
$$

2. If $n=k$, then we have

$$
B_{k}(\lambda)=\sum_{m=0}^{k}\left(\begin{array}{l}
k \\
n
\end{array}\right)(1)^{k+m}(1)_{k, \lambda}\left((1)_{k-m,-\lambda}(k-m)(-\lambda-1) B_{1}(-\lambda)+B_{k-m,-\lambda}(2)\right) .
$$

\section{Remark}

Let us assume that the probability of success in an experiment is $p$. We wondered if we could say the probability of success in the 9th trial is still $p$ after failing eight times in a ten trial experiment, because there is a psychological burden to be successful. It seems plausible that the probability is less than $p$. The degenerate Bernstein polynomial $B_{n}(x \mid \lambda)$ is used in the probability of success. Thus, we give examples in our results as follows:

Example 1. Let $n=2$, we have

$$
\begin{aligned}
B_{2}(2 \mid \lambda)= & 2(1)_{1, \lambda}(\lambda-1) B_{1}(\lambda)+B_{2}(\lambda) \\
& =2(\lambda-1)\left(-\frac{1}{2}\right)+\frac{\lambda}{2}+\frac{1}{6} \\
& =-\frac{\lambda}{2}+\frac{7}{6} .
\end{aligned}
$$

Example 2. Let $n=1$, we have

$$
\begin{aligned}
B_{1}(1-x \mid \lambda)= & (1-x)_{1, \lambda}+\sum_{m=1}^{1} B_{m, 1}(x \mid \lambda) B_{m}(\lambda) \frac{(-1)^{1-m}}{(m-1) !} \sum_{k=0}^{m-1}(-1)^{k}\left(\begin{array}{c}
m-1 \\
k
\end{array}\right) \frac{1}{x-k \lambda} \\
& =(1-x)_{1, \lambda}+B_{1,1}(x \mid \lambda) B_{1}(\lambda) \frac{1}{x} \\
& =-x+\frac{1}{2} .
\end{aligned}
$$

Example 3. Let $n=1, k=2$, we have

$$
\begin{aligned}
(x)_{1, \lambda} \sum_{m=0}^{2}\left(\begin{array}{c}
2 \\
m
\end{array}\right)(-1)^{m-2} B_{1}(1-x+m \mid \lambda) & =x\left(B_{1}(1-x \mid \lambda)-2 B_{1}(2-x \mid \lambda)+B_{1}(3-x \mid \lambda)\right) \\
& =-x\left(\left(-x+\frac{1}{2}\right)-2\left(-x+\frac{3}{2}\right)+\left(-x+\frac{5}{2}\right)\right) \\
& =0 .
\end{aligned}
$$

\section{Conclusions}

In this paper, we studied the fully degenerate Bernoulli polynomials associated with degenerate Bernstein polynomials. In Section 1, Equations (12), (18), (20) and (21) are some properties of them. In Section 2, Theorems 1-3 are results of identities for fully degenerate Bernoulli polynomials in connection with degenerate Bernstein polynomials by means of bosonic $p$-adic integrals on $\mathbb{Z}_{p}$ 
and generating functions. Theorems 4-6 are results of higher-order fully Bernoulli polynomials in connection with two variable degenerate Bernstein polynomials by means of bosonic $p$-adic integrals on $\mathbb{Z}_{p}$ and generating functions.

Author Contributions: Conceptualization, W.K. and L.-C.J.; Data curation, L.-C.J.; Formal analysis, L.-C.J.; Funding acquisition, J.G.L.; Investigation, J.G.L., W.K. and L.-C.J.; Methodology, W.K. and L.-C.J.; Project administration, L.-C.J.; Resources, L.-C.J.; Supervision, L.-C.J.; Visualization, L.-C.J.; Writing-original draft, W.K. and L.-C.J.; Writing-review \& editing, J.G.L. and L.-C.J.

Funding: This paper was supported by Wonkwang University in 2018.

Conflicts of Interest: The authors declare that they have no competing interests.

\section{References}

1. Kim, T. Barnes' type multiple degenerate Bernoulli and Euler polynomials. Appl. Math. Comput. 2015, 258, 556-564. [CrossRef]

2. Kim, D.S.; Kim, T. Identities for degenerate Bernoulli polynomials and Korobov polynomials of the first kind. Sci. China Math. 2019, 62, 999-1028. [CrossRef]

3. Kim, D.S.; Kim, H.Y.; Kim, D.J.; Kim, T. Identities of Symmetry for Type 2 Bernoulli and Euler Polynomials. Symmetry 2019, 11, 613. [CrossRef]

4. Kim, D.S.; Kim, T. Degenerate Bernstein polynomials. Rev. R. Acad. Cienc. Exactas Fís. Nat. Ser. A Mater. 2018, 1-8. [CrossRef]

5. Kim, D.S.; Kim, T. Correction to: Degenerate Bernstein polynomials. Rev. R. Acad. Cienc. Exactas Fís. Nat. Ser. A Mater. 2019, 1-2. [CrossRef]

6. Kim, D.S.; Kim, T. Some Identities on Degenerate Bernstein and Degenerate Euler Polynomials. Mathematics 2019, 7, 47. . [CrossRef]

7. Kim, T. q-Volkenborn integration. Russ. J. Math. Phys. 2002, 9, 288-299.

8. Kim, D.S.; Kim, T.; Mansour, T.; Seo, J.-J. Fully degenerate poly-Bernoulli polynomials with a $q$ parameter. Filomat 2016, 30, 1029-1035. [CrossRef]

9. Kim, D.S.; Kim, T. A Note on polyexponential and unipoly functions. Russ. J. Math. Phys. 2019, 26, 40-49. [CrossRef]

10. Kim, T.; Kim, D.S. Extended Stirling numbers of the first kind associated with Daehee numbers and polynomials. Rev. R. Acad. Cienc. Exactas Fis. Nat. Ser. A Mater. 2019, 113, 1159-1171. [CrossRef]

11. Pyo, S.-S.; Kim, T.; Rim, S.-H. Degenerate Daehee Numbers of the Third Kind. Mathematics 2018, 6, 239. [CrossRef]

12. Kim, D.S.; Kim, T.; Seo, J.-J. Fully degenerate poly-Bernoulli numbers and polynomials. Open Math. 2016, 14, 545-556. [CrossRef]

13. Kim, T.; Kwon, H.I.; Mansour, T.; Rim, S.-H. Symmetric identities for the fully degenerate Bernoulli polynomials and degenerate Euler polynomials under symmetric group of degree n. Util. Math. 2017, 103, 61-72.

14. Araci, S.; Acikgoz, M. A note on the Frobenius-Euler numbers and polynomials associated with Bernstein polynomials. Adv. Stud. Contemp. Math. (Kyungshang) 2012, 22, 399-406.

15. Bayad, A.; Kim, T. Identities involving values of Bernstein, $q$-Bernoulli, and $q$-Euler polynomials. Russ. J. Math. Phys. 2011, 18, 133-143. [CrossRef]

16. Choi, J. A note on $p$-adic integrals associated with Bernstein and $q$-Bernstein polynomials'. Adv. Stud. Contemp. Math. (Kyungshang) 2011, 21, 133-138.

17. Kim, D.S.; Kim, T.; Jang, G.W.; Kwon, J. A note on degenerate Bernstein polynomials. J. Inequal. Appl. 2019, 2019, 129. [CrossRef]

18. Kim, T.; Choi, J.; Kim, Y.H.; Ryoo, C.S. On the fermionic $p$-adic integral representation of Bernstein polynomials associated with Euler numbers and polynomials. J. Inequal. Appl. 2010, 1, 864247. [CrossRef]

19. Kim, T.; Choi, J.; Kim, Y.H. On the $k$-dimensional generalization of $q$-Bernstein polynomials. Proc. Jangjeon Math. Soc. 2011, 14, 199-207.

20. Kim, T. A note on $q$-Bernstein polynomials. Russ. J. Math. Phys. 2011, 18, 73-82. [CrossRef] 
21. Kim, T.; Kim, Y.H.; Bayad, A. A study on the $p$-adic $q$-integral representation on Z p associated with the weighted $q$-Bernstein and $q$-Bernoulli polynomials. J. Inequal. Appl. 2011, 513821. [CrossRef]

22. Kim, T.; Lee, B.; Lee, S.-H.; Rim, S.-H. A note on $q$-Bernstein polynomials associated with $p$-adic integral on $\mathbb{Z}_{p}$. J. Comput. Anal. Appl. 2013, 15, 584-592.

23. Kim, W.J.; Kim, D.S.; Kim, H.Y.; Kim, T. Some identities of degenerate Euler polynomials associated with Degenerate Bernstein polynomials. arXiv 2019, arXiv:1904.08592.

24. Kurt, V. Some relation between the Bernstein polynomials and second kind Bernoulli polynomials. Adv. Stud. Contemp. Math. (Kyungshang) 2013, 23, 43-48.

25. Ostrovska, S. On the q-Bernstein polynomials. Adv. Stud. Contemp. Math. (Kyungshang) 2005, 11, $193-204$.

26. Park, J.-W.; Pak, H.K.; Rim, S.-H.; Kim, T.; Lee, S.-H. A note on the $q$-Bernoulli numbers and $q$-Bernstein polynomials. J. Comput. Anal. Appl. 2013, 15, 722-729.

27. Siddiqui, M.A.; Agrawal, R.R.; Gupta, N. On a class of modified new Bernstein operators. Adv. Stud. Contemp. Math. (Kyungshang) 2014, 24, 97-107.

(C) 2019 by the authors. Licensee MDPI, Basel, Switzerland. This article is an open access article distributed under the terms and conditions of the Creative Commons Attribution (CC BY) license (http:// creativecommons.org/licenses/by/4.0/). 